\title{
Judicialização da Política de Assistência Farmacêutica: Discussão sobre as Causas de Pedir no Distrito Federal
}

Judicialization of Pharmaceutical Assistance Policy: discussion of causes for the actions in Federal District

\section{Simone Honorato ${ }^{1}$}

Resumo: O direito à saúde, garantido constitucionalmente, abriu a possibilidade de sua tutela judicial pelo cidadão, fazendo crescer o número de ações judiciais e gerando o fenômeno da judicialização da saúde. A pesquisa discutiu as causas de pedir verificadas nos acórdãos do Tribunal de Justiça do Distrito Federal e Territórios (TJDFT), relativos às ações impetradas contra a Secretaria de Saúde do Distrito Federal (SES/DF) para o fornecimento de medicamentos, e identificou os medicamentos pleiteados, bem como suas classes terapêuticas. Tratou-se de estudo transversal e retrospectivo de abordagem qualiquantitativa abrangendo os anos de 2010 a 2012. A amostra selecionada foi de 359 acórdãos. Foi verificado que $37,0 \%$ das ações demandavam medicamentos não elencados em listas oficiais; $24,2 \%$ motivaram-se pela falta do medicamento na SES/DF e $13,4 \%$ por interrupção no fornecimento. As classes terapêuticas mais demandadas foram: $22,8 \%$ de antineoplásicos e imunomoduladores; $22,7 \%$ para o sistema nervoso e $12 \%$ de cardiovasculares. Apesar dos esforços do Estado previstos nas políticas públicas, verificou-se que atos administrativos dos gestores do SUS impactaram diretamente na judicialização da assistência farmacêutica, resultando nas demandas judiciais por medicamento contra a SES/DF.

Palavras-chave: Assistência Farmacêutica, Judicialização da Assistência Farmacêutica, Decisões Judiciais, Sistema Único de Saúde.

Abstract: The right to health, constitutionally guaranteed, opened the possibility of judicial protection for the citizen, growing the number of lawsuits and generating the Health Judicialization phenomenon. The research discussed the causes of action observed in the judgments of the Court of Justice of the Federal District and Territories (TJDFT) related to lawsuits filed against the Federal District Health Department (SES / DF) for the supply of medicines, and identified medicines pleaded, as well as its therapeutic classes. This was cross-sectional retrospective study of qualitative and quantitative approach covering the years 2010 to 2012 . The selected sample of 359 cases. It was found that $37.0 \%$ of the shares demanded drugs not listed on official lists; $24,2 \%$ were motivated by the lack of medicine in the SES / DF and $13.4 \%$ for disruption in supply. The highest demanded therapeutic classes were: $22.8 \%$ of antineoplastic and immunomodulating agents; $22.7 \%$ to $12 \%$ nervous system and cardiovascular. Despite the efforts of the planned public policy of the state, it was found that administrative acts of SUS managers had impact directly on the judicialization of pharmaceutical care, resulting in lawsuits by drug against SES / DF.

Keywords: Pharmaceutical Assistance, Judicialization of Pharmaceutical Services, judgments, Health System.

${ }^{1}$ Departamento de Saúde Coletiva, Universidade de Brasília (UnB). Brasília, DF, Brasil. E-mail: simone.honorato@uol.com.br 
Resumen: El derecho a la salud, garantizado por la Constitución, se abrió la posibilidad de la protección judicial de los ciudadanos, al crecer el número de demandas y la generación de la legalización del fenómeno salud. La investigación analizó las causas de acción que se observan en el Tribunal de Justicia del Distrito Federal y Territorios (TJDFT) relacionados con demandas presentadas contra el Departamento de Salud del Distrito Federal (SES / DF) para el suministro de medicamentos y medicamentos identificados declarado, así como sus clases terapéuticas. Este fue un estudio retrospectivo transversal del enfoque cualitativo y cuantitativo para los años 2010 y 2012. La muestra seleccionada de 359 casos. Se encontró que $37,0 \%$ de las acciones exigió drogas no mencionadas en las listas oficiales; $24,2 \%$ motivado por la falta de medicamentos en el SES / DF y el $13,4 \%$ para la interrupción en el suministro. Las más altas clases terapéuticas de demanda fueron: $22,8 \%$ de los agentes antineoplásicos e inmunomoduladores; $22,7 \%$ a $12 \%$ del sistema nervioso y cardiovascular. A pesar de la política pública prevista de los esfuerzos estatales, se encontró que los actos administrativos de los gestores del SUS impactados directamente sobre la legalización de la atención farmacéutica, lo que resulta en demandas por drogas contra SES / DF.

Palabras-Ilave: Asistencia Farmacéutica, Legalización de Servicios Farmacéuticos, juicios, Sistema de Salud

\section{Introdução}

Os princípios que nortearam o chamado Movimento da Reforma Sanitária no Brasil foram contemplados no texto da Constituição Federal de 1988 (1), que inseriu a saúde, no art. 196, como direito social universal, integral, descentralizado, acessível, democrático, garantido mediante políticas sociais e econômicas que visem à redução do risco de doença e de outros agravos e ao acesso universal e igualitário às ações e serviços para sua promoção, proteção e recuperação.

Conforme disposto na Carta Magna, em seu art. 50 - $\S 1^{\circ}$ "As normas definidoras dos direitos e garantias fundamentais tem aplicação imediata" e se prestam a obrigar que 0 Estado tome medidas para melhorar as condições sociais dos cidadãos, no sentido de diminuir as desigualdades sociais.

Sant"Ana et al. (2) enfatizam que a positivação do direito à saúde ocorrida no Brasil trouxe como repercussão mais imediata da reforma no sistema o estabelecimento de uma nova relação jurídica entre o Estado e seus cidadãos, que adquiriram direitos sociais e subjetivos, abrindo-se a possibilidade de tutela judicial do direito à saúde. No entanto, no âmbito da assistência farmacêutica pública, apesar dos esforços do Estado previstos nas políticas relacionadas ao tema, estudos demonstram que ainda há falhas no sistema para 
Cad. Ibero-Amer. Dir. Sanit., Brasília, v.4, n.3, jul./set. 2015 ISSN 2358-1824

atender a demanda da população em relação ao fornecimento de medicamentos.

O início do movimento de judicialização da saúde e da assistência farmacêutica no Brasil se deu com os pacientes da Síndrome da Imunodeficiência Adquirida (AIDS). A articulação desse grupo de pacientes resultou na promulgação da Lei $n^{0}$ 9.313, de 13 de novembro de 1996 (3), que instituiu a distribuição gratuita de medicamentos, pelo SUS, para os portadores do vírus da imunodeficiência humana (HIV). A partir da incorporação dos medicamentos dedicados ao tratamento dessa moléstia ao elenco do SUS, a demanda pela via judicial por esses medicamentos diminuiu, porém a partir desse momento outras demandas começaram a surgir. Em estudos recentes no Rio de Janeiro, a demanda relacionada ao HIV/AIDS continua muito baixa, inferior a $5 \%$.

Nesse cenário, a via Judicial representa ao cidadão a possibilidade de acesso integral à saúde, fundamentado na norma constitucional, seja para tratamentos, diagnósticos, realização de exames, internações em UTI ou acesso aos medicamentos.

Segundo um estudo de 2005 no estado de São Paulo, 62\% dos medicamentos demandados judicialmente faziam parte de listas de medicamentos de programas do SUS. Já em 2006, no mesmo estado, outro estudo apontou que a maioria (77\%) das ações ajuizadas contra a Secretaria de Saúde demandava acesso a produtos e serviços não previstos nos protocolos e programas executados pelo SUS naquele estado. No Distrito Federal, achados demonstraram que $47 \%$ dos medicamentos pleiteados pela via judicial de 1997 até 2005, faziam parte da Relação Nacional de Medicamentos (RENAME).

Nesse contexto, Sant'Ana et al. (1) destacam que a judicialização da assistência farmacêutica isoladamente não é suficiente para reduzir as desigualdades em saúde. No entanto, a atuação do Poder Judiciário nessas demandas possibilita o acesso imediato a um direito constitucional e provoca os gestores de saúde a adequar os programas às necessidades da população.

Identifica-se a tendência crescente de envolvimento do Poder Judiciário, antes um Poder periférico inacessível aos leigos, com a questão social, agora se mostrando uma instituição central à democracia brasileira, tanto na política quanto no âmbito social, tornando-o mais presente e aumentando sua visibilidade na mídia e em pesquisas universitárias. 
Objetivou-se nesse estudo, identificar as causas de pedir que motivaram as ações contra a Fazenda Pública do Distrito Federal entre 2010 e 2012 e discutir as situações que levaram os pacientes a buscarem a via judicial para obter o fornecimento de medicamentos pelo Sistema Único de Saúde, verificando a tendência decisória do Tribunal de Justiça do Distrito Federal e Territórios (TJDFT). Igualmente buscou-se identificar o rol dos medicamentos pleiteados e suas classes terapêuticas, bem como os componentes mais demandados.

\section{Método}

Tratou-se de estudo de corte transversal descritivo retrospectivo, limitado aos anos de 2010 a 2012. A pesquisa teve caráter jurisprudencial, com abordagem quali-quantitativa por análise de conteúdo. Os dados foram coletados no banco eletrônico do Tribunal de Justiça do Distrito Federal e Territórios (TJDFT) e foi realizada a análise das demandas judiciais que alcançaram a segunda instância de julgamento, sendo escolhidos como unidades de análise, os acórdãos emitidos pelo colegiado do TJDFT. Os objetos de análise foram as decisões colegiadas (acórdãos) do Tribunal de Justiça do Distrito Federal e Territórios referentes às ações impetradas por indivíduos contra o Secretário de Saúde do Distrito Federal, requerendo o fornecimento de medicamentos pelo Sistema Único de Saúde (SUS).

Iniciou-se a pesquisa com a busca pelo descritor "medicamento", na base de dados públicos do TJDFT. Foram incluídos acórdãos referentes, exclusivamente, ao fornecimento de medicamentos pelo Sistema Único de Saúde, e foram excluídos aqueles referentes a demandas por procedimentos cirúrgicos, por exames de diagnósticos, por internações em unidades de terapia intensiva, entre outros pedidos.

Após a seleção, foi realizada a leitura flutuante de todos os acórdãos e foi realizada a codificação dos dados brutos, buscando identificar sistematicamente as unidades de registro que definissem o tema das categorias. Utilizou-se exclusivamente o texto descrito no acórdão, assim, um acórdão que citava claramente a demanda direta ao judiciário, foi enquadrado nessa categoria, independente do detalhamento de qual medicamento se tratava e se o mesmo estava nas listas do Sistema. Desse modo surgiram sete categorias pautas nas causas de pedir do demandanete, que motivaram seu ingresso no Sistema 
Judiciário, detalhadas no Quadro 1.

Quadro 1 - Categorias de análise das causas de pedir nos mandados de segurança do TJDFT - 2010/2012

\begin{tabular}{|c|c|c|}
\hline \multicolumn{2}{|r|}{ Nome da Categoria } & Resumo da categoria \\
\hline 1 & $\begin{array}{l}\text { Solicitação } \\
\text { Indeferida }\end{array}$ & $\begin{array}{l}\text { Casos em que o medicamento estava presente em listas oficiais } \\
\text { do SUS, porém o pedido administrativo foi indeferido } \\
\text { formalmente. }\end{array}$ \\
\hline 2 & $\begin{array}{ll}\text { Solicitação } & \text { Administrativa } \\
& \text { Não Respondida }\end{array}$ & $\begin{array}{l}\text { Casos em que o medicamento não estava elencado nas listas } \\
\text { do SUS, porém o paciente entrou com pedido de fornecimento } \\
\text { pela via administrativa e não obteve nenhuma resposta. }\end{array}$ \\
\hline 3 & Demanda Direta ao Judiciário & $\begin{array}{l}\text { Casos em que o paciente entrou com mandado direto no } \\
\text { Sistema Judiciário sem procurar a via administrativa } \\
\text { anteriormente. }\end{array}$ \\
\hline 4 & Falta do Medicamento & $\begin{array}{l}\text { Casos em que o medicamento estava elencado no SUS, porém } \\
\text { não havia disponibilidade no estoque da SES/DF. }\end{array}$ \\
\hline 5 & $\begin{array}{ll}\text { Fornecimento } & \text { Interrompido } \\
& \text { ou intermitente }\end{array}$ & $\begin{array}{l}\text { Casos em que os pacientes que estavam em tratamento } \\
\text { pararam de receber o medicamento temporária ou } \\
\text { definitivamente por decisão administrativa. }\end{array}$ \\
\hline 6 & $\begin{array}{l}\text { Medicamento não Elencado em } \\
\text { Listas Oficiais }\end{array}$ & $\begin{array}{l}\text { Casos em que o medicamento não constava em listas oficiais do } \\
\text { SUS. }\end{array}$ \\
\hline 7 & $\begin{array}{l}\text { Medicamento Não Registrado no } \\
\text { Brasil }\end{array}$ & $\begin{array}{l}\text { Casos em que o medicamento não possuía autorização de } \\
\text { comercialização concedida pelo órgão competente no Brasil, no } \\
\text { caso atual a Agência Nacional de Vigilância Sanitária (ANVISA). }\end{array}$ \\
\hline
\end{tabular}

Fonte: Acórdãos coletados do site do TFDFT.

O estudo apresentou algumas limitações sendo a principal delas a impossibilidade de consulta física à petição inicial e documentos originais como receituários médicos e laudos de exames. Todas as informações utilizadas nessa pesquisa foram retiradas dos acórdãos publicados pelo colegiado do TJDFT, no banco de dados eletrônico.

Para a confirmação da presença do medicamento em listas oficiais da assistência farmacêutica no Distrito Federal (REME/DF), foram consideradas as listas disponíveis no sítio eletrônico da Secretaria de Atenção à Saúde do Distrito Federal (SAS/DF), vigentes entre 2010 e 2012, referentes à atenção básica, componente especializado e medicamentos estratégicos. No entanto, verificou-se que muitas das ações haviam sido iniciadas em anos anteriores ao período do estudo, de modo que a amostra continha pleitos iniciados desde 2004. Para esses casos foram pesquisadas as RENAME vigentes à época da propositura da ação. 
Os medicamentos encontrados foram classificados de acordo com o Sistema Anatômico Terapêutico Químico ATC (Anatomical Therapeutic Chemical) (4), que desde 1996, passou a ser reconhecido pela Organização Mundial de Saúde (OMS) como padrão internacional para os estudos de utilização de medicamentos (EUM). Para esse estudo utilizou-se o primeiro nível da classificação no sistema de classificação ATC, no qual os fármacos são divididos em 14 diferentes grupos, de acordo com o órgão ou sistema no qual eles atuam. Apenas para os fármacos classificados no grupo L (Agentes Antineoplásicos), estendeu-se a classificação até o segundo nível, diferenciando-se os antineoplásicos e os imunossupressores.

O Componente da Assistência Farmacêutica foi identificado no elenco de medicamentos pela verificação da presença dos mesmos nas listas oficiais do SUS.

O projeto de pesquisa foi submetido à apreciação do Comitê de Ética em Pesquisa com Seres Humanos da Faculdade de Ciências Saúde da Universidade de Brasília (CEPFS/UnB). Tendo em vista que o estudo não envolveu diretamente seres humanos e utilizou de base de dados pública, o referido CEP opinou pela aprovação do projeto e dispensa do Termo de Consentimento Livre e Esclarecido (TCLE).

\section{Resultados}

Após a análise das causas de pedir nos 359 acórdãos, foram elaboradas sete categorias relacionadas aos motivos do ingresso do demandante no sistema judiciário, sendo elas: "Solicitação Administrativa Indeferida", "Solicitação Administrativa Não Respondida", "Demanda direta ao Judiciário", "Falta do Medicamento", "Fornecimento Interrompido ou intermitente", "Medicamento não elencado em Listas Oficiais", "Medicamento Não Registrado no Brasil".

A busca inicial pelo descritor "medicamento" alcançou um retorno de 1614 acórdãos entre os anos de 2010 e 2012. Desses, foram excluídos os que eram referentes a demandas por procedimentos cirúrgicos, por exames de diagnósticos, por internações em unidades de terapia intensiva entre outros pedidos. Desse modo, foram considerados para análise 359 acórdãos no total, sendo 118 em 2010, 141 em 2011 e 100 do ano de 2012. O resultado da categorização está disposto na Tabela 1. 
Tabela 1 - Frequência das Causas de Pedir nos acórdãos do TJDFT entre 2010 e 2012

\begin{tabular}{lccccc}
\hline \multirow{2}{*}{$\begin{array}{l}\text { CATEGORIAS DE ANÁLISE } \\
\text { Causas de Pedir }\end{array}$} & 2010 & 2011 & \multicolumn{2}{c}{2012} & \\
\cline { 2 - 6 } & $\mathrm{N}(\%)$ & $\mathrm{N}(\%)$ & $\mathrm{N}(\%)$ & TOTAL N & $\%$ \\
\hline Solicitação Administrativa & $8(6,8 \%)$ & $4(2,8 \%)$ & $8(8,0 \%)$ & 20 & $(5,57 \%)$ \\
Solicitação Administrativa Não & $15(12,7 \%)$ & $25(17,7 \%)$ & $11(11,0 \%)$ & 51 & $(14,2 \%)$ \\
Demanda Direta ao Judiciário & $4(3,4 \%)$ & $4(2,80 \%)$ & 0 & 8 & $(2,2 \%)$ \\
Falta do Medicamento & $34(28,8 \%)$ & $33(23,4 \%)$ & $20(20,0 \%)$ & 87 & $(24,2 \%)$ \\
Fornecimento Interrompido ou & $1(0,80 \%)$ & $21(14,9 \%)$ & $26(26,0 \%)$ & 48 & $(13,4 \%)$ \\
Medicamento não Elencado & $54(45,8 \%)$ & $49(34,8 \%)$ & $30(30,0 \%)$ & 133 & $(37,0 \%)$ \\
Medicamento Não Registrado & $2(1,7 \%)$ & $5(3,5 \%)$ & $5(5,0 \%)$ & 12 & $(3,3 \%)$ \\
\hline TOTAL DE ACÓRDÃOS & $118(100 \%)$ & $141(100 \%)$ & $100(100 \%)$ & 359 & $(100 \%)$
\end{tabular}

Fonte: Acórdãos coletados no site do TJDFT, 2010-2012.

$\mathrm{Na}$ análise estratificada da amostra, considerando apenas Mandados de Segurança $(n=181)$, a segurança foi concedida em pouco mais de noventa por cento dos casos e houve a negativa pelo colegiado do TJDFT em quase quatro por cento. Além disso, a concessão parcial da segurança esteve presente em 4,4\% dos acórdãos, na maioria das vezes relacionada ao período de validade do mandado, que variou entre 30 dias, seis meses a um ano.

Conforme a classificação no nível 1 do código ATC, no total de frequência dos medicamentos identificados $(n=534), 22,8 \%(n=122)$ eram Agentes Antineoplásicos e Imunomoduladores (Grupo L), sendo que desses, 50\% ( $n=61)$ foram classificados no subgrupo L01 como antineoplásicos e 28,7\% ( $\mathrm{n}=35)$ no subgrupo L04 como imunossupressores; 22,7\% $(n=121)$ eram do Sistema Nervoso (grupo N); 12 \% ( $n=64)$ eram do Aparelho Cardiovascular (grupo C). A distribuição nas classes terapêuticas encontradas encontra-se na Tabela 2. 
Tabela 2 - Frequência de distribuição dos medicamentos de acordo com o $1^{\circ}$ nível da classificação Anatômico Terapêutico ATC, nos acórdãos do Tribunal de Justiça do Distrito Federal e Territórios entre janeiro de 2010 e dezembro de 2012.

\begin{tabular}{l|r|c}
\hline Grupos Anatômicos Terapêuticos & N & Frequência (\%) \\
10 nível ATC & 122 & 22,8 \\
\hline (L) Agentes antineoplásicos e imunomoduladores & 121 & 22,7 \\
(N) Sistema nervoso central & 64 & 12,0 \\
(C) Aparelho cardiovascular & 62 & 11,6 \\
(B) Sangue e órgãos hematopoiéticos & 48 & 9,0 \\
(A) Aparelho digestivo e metabolismo & 27 & 5,1 \\
(J) Anti-infecciosos gerais para uso sistémico & 24 & 4,5 \\
(M) Sistema musculoesquelético & 21 & 3,9 \\
(G) Aparelho genito-urinário e hormônios sexuais & 18 & 3,4 \\
(H) Hormônios sistémicos, excluindo hormônios sexuais e insulinas & 16 & 3,0 \\
(S) Órgãos Sensoriais - oftálmicos & 7 & 1,3 \\
(R) Aparelho respiratório & 2 & 0,4 \\
N/A - fitoterápicos & 1 & 0,2 \\
(D) Medicamentos dermatológicos & \multicolumn{2}{|c}{0,2} \\
(P) Antiparasitários & 534 & 100 \\
\hline Total & \multicolumn{2}{|c}{} \\
\hline
\end{tabular}

Fonte: Acórdãos coletados no site do TJDFT, 2010-2012.

Uma das dificuldades para agrupar as ações estudadas nos respectivos Componentes da Assistência Farmacêutica foi o fato de que as prescrições médicas podiam conter mais de um medicamento, pertencentes a diferentes Componentes, porém a demanda judicial ter sido motivada por problemas no fornecimento de apenas um dos itens do receituário. Observou-se nessa amostra que os medicamentos do Componente Básico normalmente não se mostraram alvo principal das demandas, apesar do receituário ser demandado por completo.

Em relação à presença dos medicamentos nas listas oficiais da assistência farmacêutica no SUS, verificou-se que dos 534 medicamentos identificados, 47,4\% $(n=253)$ não estavam presentes em nenhum componente da assistência e 13,1\% $(\mathrm{n}=70)$ corresponderam a antineoplásicos. No Componente Básico corresponderam 13,5\% ( $n=72)$, no Componente Especializado foram 20\% ( $n=107)$ e no Componente Estratégico foram 1,9\% $(n=10)$. Exclusivo para atendimento hospitalar e outros programas $1,9 \%(n=10)$ e medicamentos sem registro totalizaram $2,2 \%(n=12)$. 
A distribuição por Componentes da Assistência, no entanto, evidenciou que o Componente Especializado continua sendo o principal foco de demandas judiciais dentre os medicamentos elencados.

\section{Discussão}

Macedo et al. (5) enfatizam que é preciso identificar as razões que motivam cada ação, de modo que as decisões estejam amparadas por pleno conhecimento das políticas públicas de saúde, das listas de medicamentos de fornecimento público e das alternativas disponíveis no Sistema Único de Saúde, dos protocolos e restrições ao uso dos medicamentos.

O estudo atual evidenciou uma mudança discreta no padrão decisório do colegiado, uma vez que as concessões não foram sempre em favor do autorpaciente, como verificado em outros estudos, por exemplo, em 2008 no Distrito Federal verificou-se que em todas as ações estudadas, o provimento era em favor do autor-paciente. Em 2006 no estado do Rio de Janeiro, outro estudo relatou que 100\% dos processos obtiveram liminarmente seus pleitos e apenas três sentenças finais foram negadas. No caso das Apelações estudadas, houve ainda dois indeferimentos nos pedidos de tutela antecipada e sentenças finais negadas, relacionadas a casos de pedidos de medicamentos sem registro no país.

Dentre as categorias de análise das causas de pedir elaboradas nesse estudo, percebe-se que os demandantes recorrem ao sistema judiciário majoritariamente após alguma insuficiência administrativa, ou seja, as demandas diretas ao sistema, sem antes ter passado pelo trâmite administrativo para a obtenção do medicamento aparecem em apenas oito dos casos estudados.

Os casos de solicitação de medicamentos não presentes em listas oficiais se mostraram a maior causa de não prestação da assistência farmacêutica pela Secretaria de Saúde do Distrito Federal, representando 37\% do total de acórdãos estudados e apesar de frequente, estudos realizados em Minas Gerais e em São Paulo apontaram que mais da metade das demandas nesses estados, $56 \%$ e $77 \%$ respectivamente, são por medicamentos não elencados em listas oficiais. As solicitações avaliadas como improcedentes pela Secretaria de Saúde que resultaram em indeferimento formal dos pleitos apareceram numa frequência de 3,3\% dos casos no DF. 
Conforme observado por Romero (6), a ausência de explicações detalhadas sobre os critérios técnicos e fundamentos dos protocolos clínicos e das listas de medicamentos nas peças recursais e de defesa são mal recebidas pelos julgadores.

A insuficiência no abastecimento dos medicamentos elencados na Secretaria de Saúde do DF aparece em seguida como motivação das ações nos anos de 2010 e 2011 e o fornecimento intermitente ou mesmo interrompido, mostra-se expressivo já em 2011, tendo se tornado a segunda causa maior de demandas em 2012, por conta da decisão administrativa de interromper o fornecimento do Concentrado de Fator VIII de Origem Recombinante, utilizado no tratamento de hemofilia $A$ grave, por não apresentar evidência de superioridade em relação ao tratamento com o mesmo fator de origem plasmática.

A frequência de demandas por medicamentos não registrados no país parece não ser o foco das demandas no presente estudo, pois representou 3,3\% das ações, mostrando inclusive frequência menor do que a encontrada em outro estudo realizado em Minas Gerais, que chegou a registrar $5 \%$ desse tipo de pedido.

A alta frequência de pedidos por medicamentos antineoplásicos chama a atenção pela presença em $22,8 \%$ dos casos no DF, uma vez que o fornecimento desse tipo de terapia segue políticas e diretrizes próprias, por meio dos Centros de Alta Complexidade em Oncologia (CACON). Outras pesquisas discutem essa situação em que o alto custo desses medicamentos evidenciaria a forte distorção que as ações judiciais estão causando ao SUS por retirarem recursos para a compra de medicamentos pertencentes a outros componentes da assistência farmacêutica e forçarem a incorporação de novas tecnologias.

\section{Considerações Finais}

Os casos judicializados estudados demonstraram que não inclusão de medicamentos em listas oficiais e as falhas no abastecimento dos medicamentos elencados nas listas oficiais foram as maiores motivações para as ações contra o Distrito Federal entre os anos de 2010 e 2012, seja devido à indisponibilidade nos estoques ou mesmo à descontinuidade no fornecimento dos medicamentos.

Verificou-se que atos administrativos dos gestores do SUS impactam diretamente na judicialização da assistência farmacêutica, por exemplo, a decisão de suspender o fornecimento do Fator de Coagulação VIII recombinante aos portadores 
Cad. Ibero-Amer. Dir. Sanit., Brasília, v.4, n.3, jul./set. 2015 ISSN 2358-1824

de Hemofilia A no Distrito Federal, teve como consequência o aumento imediato das demandas judiciais por esse produto.

Algumas mudanças no padrão de julgamento, apontadas no estudo atual, evidenciadas com a menor frequência da concessão da segurança nos caso dos mandados julgados, aponta que o judiciário pode estar discutindo o mérito da judicialização da saúde com mais propriedade.

No entanto a efetivação do direito à saúde apresenta-se garantido pelo sistema judiciário, mesmo na existência de políticas públicas e diretrizes específicas, como no caso dos medicamentos antineoplásicos, que alerta para o desconhecimento do judiciário quanto à prestação dessa terapêutica pelo SUS.

Após avaliação dos acórdãos do estudo, infere-se que o reconhecimento do direito a assistência farmacêutica pela população ainda não está consolidado, uma vez que nos casos de ausência de resposta ao demandante após a solicitação de medicamentos pela via administrativa, os mesmos não geraram demandas imediatas ao judiciário, muitas vezes os cidadãos relataram esperar meses até entrar no sistema judiciário, ou seja, a expectativa da população é de que o poder executivo se organize para a prestação da assistência farmacêutica.

Observou-se, ainda, neste estudo, que o argumento da ausência dos medicamentos em listas oficiais não foi levado em consideração pelos julgadores para a negativa no fornecimento do medicamento, pois em suas argumentações, estes consideravam que as listas de medicamentos elencados se prestam a orientar a assistência farmacêutica, mas nunca a limitá-la. Nesse contexto, a compreensão da dinâmica entre a política pública, os operadores do direito e os usuários do sistema é essencial para efetividade do direito à saúde e para a ampliação do acesso à justiça e à saúde.

\section{Referências Bibliográficas}

1 Brasil. Constituição da República Federativa do Brasil de 1988. [Internet] Brasília. [Acesso em 17 ago 2013]. Disponível em: http://www.planalto.gov.br/ccivil 03/constituicao/constituicaocompilado.htm

2 Sant'ana JMB et al. Essencialidade e assistência farmacêutica: considerações sobre o acesso a medicamentos mediante ações judiciais no Brasil. Revista 
Panamericana de Salud Publica [Internet]. 2011 [Acesso em 20 de maio 2013], 29(2): 138-144. Disponível em http://www.scielosp.org/pdf/rpsp/v29n2/a10v29n2.pdf

3 Brasil. Lei no 9.313, de 13 de novembro de 1996. Dispõe sobre a distribuição gratuita de medicamentos aos portadores do HIV e doentes de AIDS. Diário Oficial da União [Internet] Brasília, 14 nov 1996. [Acesso em 24 mar 2014]. Disponível em: http://www.planalto.gov.br/ccivil 03/leis/19313.htm

4 WHO Collaborating Centre for Drug Statistics Methodology, Guidelines for ATC classification and DDD assignment, 2013. Oslo, 2012. [Acesso em 18 mai 2013]. Disponível em: http://www.whocc.no/filearchive/publications/1 2013guidelines.pdf

5 Macedo El de, Lopes LC, Barberato-Filho S. Análise técnica para a tomada de decisão do fornecimento de medicamentos pela via judicial. Revista de Saúde Pública [Internet]. 2011 [Acesso em 24 mar 2014], 45(4): 706-713. Disponível em http://www.scielo.br/pdf/rsp/v45n4/2603.pdf

6 Romero LC. A jurisprudência do Tribunal de Justiça do Distrito Federal em ações de medicamentos. Revista de Direito Sanitário [Internet]. 2010 [Acesso em 20 de maio 2013], 11(2): 11-59. Disponível em http://www.revistas.usp.br/rdisan/article/view/13207. 